in Escherichia coli have proved less effective than existing vaccines. Thus, even after two doses, antibody levels are much lower than after a single dose of conventional whole-virus vaccines. Synthetic peptides are reported by Bittle et al. to be far more effective than VP1 but this comparison is a bit unreal for two reasons: first, the VP1 they used was prepared by SDS treatment and polyacrylamide gel electrophoresis which is a denaturing step and may hide the sites in VP1 reacting with antipeptide sera or with native virions. Also, VP1 is a relatively small protein, whereas the synthetic peptide was coupled to KLH, one of the most immunogenic carrier proteins in the immunologist's repertoire. The results of coupling VP1 produced by genetic engineering to a similar immunogenic carrier would have been a more valid comparison. Perhaps the most encouraging feature, however, was the almost equally good results they obtained from a single dose using aluminium hydroxide compared with Freunds adjuvant. The dose schedule and duration of immunity are important aspects of vaccine development. As noted earlier for foot-and-mouth disease vaccine, because of the difficulty of mustering animals for vaccination, protection is required from a single dose, but two to three doses may be given each year. For many other vaccines a two-dose primary schedule can be followed by booster doses over a period of years. The performance of vaccines based on synthetic peptides in these respects will need study, but there is no reason to suppose they will prove less efficient than existing killed vaccines.

The feasibility and economics of synthetic peptide vaccines are difficult to judge. The situation for foot-and-mouth disease, where there is an existing relatively cheap vaccine, is very different from that of hepatitis B, for example, where the vaccine is very expensive and can be made only on a limited scale. Another key factor will be the degree of purity of the peptides required. Lerner and his colleagues did not make any special efforts to purify the peptides that are produced by the Merriefield solid-phase synthesis. Plainly it will be essential to establish that the effects are due to a peptide truly of the sequence deduced from DNA sequencing. The degree of purity ultimately required will doubtless vary according to the application. It will be necessary to have a synthetic process that consistently produces the same product no doubt the spur of a practical application will lead to improved methods and reduced

1. Kleid, D.G. et al. Science 214, 1125 (1981)

2. Langbeheim, H., Arnon, R.\& Sela, M. Proc. natn. Acad. Sci. U.S.A. 73, 4636 (1976)

3. Audibert, F. et al. Nature 289, 593 (1981)

4. Beachey, E. H. et al. Nature 292, 452 (1981).

5. Dreesman, G.R. et al. Nature 295, 158 (1982).

6. Lerner, R.A. et al. Proc, natn. Acad. Sci. U.S.A. 78. 3403 (1981).

Wiley, D.C., Wilson, I.H. \& Skehel, J.J. Nature 289, 373 (1981).

Green, N. et al. Cell 28, 477 (1982)

9. King, A.M.Q. et al. Nature 293, 479 (1981). costs. The size of the peptide to be made will be a key factor. Another important element requiring more research is the carrier. In the present experiments with FMDV, KLH was used but it seems certain to be superseded because of supply problems. The addition of a carrier such as KLH negates some of the advantages of a defined synthetic vaccine and problems of hypersensitivity to the carrier may occur when repeated injections are necessary. Clearly there are alternative carriers, such as tetanus toxoid or poly-DL-lysine, and this is an important matter for more research.
Similarly, there will be a need for an adjuvant. Aluminium hydroxide is a clinically acceptable adjuvant for man and it is encouraging that it worked for FMDV. The question of the adjuvant is linked to the requirement for a carrier and the best combination will need to be reassessed. Knowledge about adjuvants is at present a dark area of immunology.

The work of Bittle et al. is a significant step along the road to synthetic vaccines. It opens up exciting possibilities for vaccine development which should be vigorously pursued.

\title{
The internal evolution of Venus and the galilean satellites
}

\section{from Sean C. Solomon}

THE solid planets and satellites of this Solar System display a great diversity of surface features. The Moon and Mercury and many of the icy satellites of Jupiter and Saturn have heavily cratered terrains that have been little altered for billions of years. Jupiter's moon Io has been continuously resurfaced over periods of millions of years by vigorous volcanic eruptions fuelled by the dissipation of huge solid-body tides. The Earth has a lithosphere underlying the ocean basins that is recycled by plate tectonics over a period of about 100 million years. Despite their differences, all these bodies have been shaped by common physical processes during their formation and subsequent evolution. A continuing challenge, made evident by the many new results on the internal and surface evolution of planets and satellites presented at the 13th Lunar and Planetary Science Conference*, is to understand how these common processes have produced the observed diversity of Solar System objects.

Particular interest at the conference was focused on Venus and the galilean satellites of Jupiter, all objects of recent scrutiny by US and Soviet spacecraft.

Dramatic new results have come from Venera 13 and 14 which landed on Venus on 1 and 15 March respectively (V.L. Barsukov and $\mathrm{Yu}$. A. Surkov, Vernadsky Institute of Geochemistry and Analytical Chemistry; and see News and Views 296, $607 ; 1982)$. Most notable are the chemical analyses of compositions of Venus soils, derived by $X$-ray fluorescence, that indicate similarities to a high-potassium alkali basalt at Venera 13 and a terrestrial oceanic tholeiite at Venera 14. On Earth tholeiites, because they comprise the upper igneous crust of all ocean basins, are the most common volcanic rock type.

*The 13th Lunar and Planetary Science Conference was held at the Lyndon B. Johnson Space Center, Houston, Texas on 15-19 March 1982.
Discovery of such basalts on Venus supports the hypothesis that the majorelement compositions of the two planets and their respective mechanisms for the formation and eruption of basaltic magma are similar.

Detailed comparisons of Venus and Earth with respect to planetary composition, mechanical properties and tectonic evolution were also presented. The bulk density of Venus, after correcting for the effects of compression, is generally estimated to be about one per cent less than that of the Earth ${ }^{1}$. The difference is often explained in terms of a slightly different composition for the two planets ${ }^{1,2}$. K.A. Goettel (Washington University) suggested that Venus may have a bulk density at standard conditions equal to or greater than that of the Earth. Such a result would hold if internal temperatures are significantly higher for Venus than for Earth at comparable depths and if Venus has a thicker layer of basaltic crust ${ }^{3}$. The required higher temperatures may occur, however, only if Venus has no analogue to terrestrial plate recycling ${ }^{4}$. If Goettel's suggestion is correct, then cosmochemical models for inner planet formation will require revision.

There is much debate over whether some version of plate tectonics operates on Venus. The issue has broad implications for our understanding of heat transfer and surface evolution on silicate planets. $H$. Spetzler, I.C. Getting (CIRES, University of Colorado) and H. Mizutani (University of Nagoya) have made a new contribution in a theoretical formulation for brittle failure in silicate planets based on stress corrosion theory and the interaction of growing cracks. Including the effects of

Sean C. Solomon is an Associate Professor of Geophysics in the Department of Earth and Planetary Sciences, Massachusetts Institute of Technology, Cambridge, Massachusetts 02139.

(c) 1982 Macmillan Journals Ltd 
watcr on the rate of crack growth, they have calculated the effective failure strength as a function of depth in each of the terrestrial planets. For Venus, assuming the partial pressure of $\mathrm{H}_{2} \mathrm{O}$ in near-surface rocks to equal that in the lower atmosphere, they calculate a very low value of crustal strength (about $10 \mathrm{MPa}$ ) and conclude that plate subduction on Venus would be unlikely.

This author and J.W. Head (Brown University) took a different view. Venus must lose an amount of heat per mass roughly comparable to that known for the Earth $^{5}$. Solid planets and satellites transfer internally generated heat to the surface by plate recycling, as on Earth ${ }^{6}$, lithospheric conduction, as on the Moon, and hotspot volcanism, as on Io. Each has been suggested as the dominant mechanism for lithospheric heat transfer on Venus ${ }^{6-8}$ and we argued that none can be ruled out given our present knowledge of the venusian surface. In particular, plate recycling cannot be excluded once it is recognized that the characteristics of plate divergence and convergence zones on Venus and Earth will be different because of the different surface temperatures ( $740 \mathrm{~K}$ on Venus) and surface abundances of water. Whatever the dominant mechanism of lithospheric heat transfer, however, given a global heat loss per mass comparable to that for the Earth, we must conclude that most of the largescale topographical features on Venus are young compared with the surface ages of the smaller terrestrial planets.

The galilean satellites, because their surfaces are cold and are composed of unusual materials, display landforms and surface histories that are difficult to understand simply by extrapolation from the known outcomes of processes on the terrestrial planets. Europa, for instance, has a high-albedo icy surface criss-crossed by numerous darker lineations of great length and probably of tectonic origin ${ }^{9}$. T.B. McCord (University of Hawaii) and colleagues reported on a new spectral unit map of Europa derived from Voyager multispectral images. At least four distinguishable units occur; the darker lineations are spectrally similar to other

1. Ringwood, A.E. \& Anderson, D.L. Icarus 30, 243-253 (1977).

2. Goettel, K.A., Shields, J.A. \& Decker, D.A. Proc. lunar planet. Sci. 12B, 1507-1516 (1981)

3. Anderson, D.L. Geophys. Res. Lett. 7, 10t-102 (1980).

4. Turcotte, D.L., Cooke, F.A. \& Willemann, R.J. Proc. Turcotte, D.L., Cooke, F.A. \& Willemann, R.J.
IOth lunar planet. Sci. Conf., 2375-2392 (1979).

5. Sclater, J.G., Jaupart, C. \& Galson, D. Rev. Geophys. Space Phys. 18, 269-312 (1980).

6. Phillips, R.J. et al. Science 212, 879-887 (1981).

7. Head, J.W., Yuter, S.E. \& Solomon, S.C. Am. Scient. 69, 614-623 (1981)

8. Phillips, R.J. \& Malin, M.C. in Venus (eds Hunten, D.M., Colin, L. \& Donahue, T.M.) (University of Arizona Press, in the press).

9. Smith, B.A. et al. Science 204, 951-972 (1979); 206, 927-950 (1979).

10. Helfenstein, P. \& Parmentier, E.M. Proc. Ilth lunar planet. Sci. Conf., 1987-1998 (1980)

1. McKinnon, W.B. Proc. lunar planet. Sci. 12B, 1585-1598 (1981)

12. Smith, B.A. et al. Science 212, 163-191 (1981); 215 , 504-537 (1982). dark units. E. Schonfeld (Johnson Space Center) proposed that the brown colour of the lineations and the low reflectance of the satellite at 3-4 $\mu \mathrm{m}$ may be due to the presence of organic molecules erupted from Europa's interior during episodes of aqueous volcanism. Interpreting the lineations as lithospheric fractures, $P$. Helfenstein (Brown University) showed that they may be the result of tidal stresses, although the details of such an explanation are considerably more complicated than in earlier versions ${ }^{10}$.

The galilean satellites Ganymede and Callisto are similar in size and bulk density, yet their surfaces differ markedly ${ }^{9}$. Callisto displays a surface that has been little altered since an era of heavy impact bombardment. Ganymede, however, preserves heavily cratered units but also contains younger and more lightly grooved terrain, thought to be of extensional tectonic origin, suggesting the hypothesis that Ganymede has undergone greater global expansion than Callisto since the period of high impact flux. P.Y. Huang (MIT) and this author tested this idea by calculating the lithospheric thermal stress that would accumulate from global thermal evolution, including the effects of subsolidus convection and phase changes in $\mathrm{H}_{2} \mathrm{O}$ ice, and concluded that Ganymede and Callisto would have had similar stress histories if their initial conditions were similar. If, however, Ganymede began at higher temperatures because of thermal gradients in the proto-jovian nebula ${ }^{11}$, the difference in stress histories can account for the different surface characteristics. New estimates of lithospheric thermal gradients versus time in Ganymede and Callisto were reported by Q.R. Passey (Caltech) and E.M. Shoemaker (US Geological Survey) from the distribution of viscously relaxed craters of various diameters and by W.B. McKinnon (University of Arizona) from the widths of concentric ring scarps, interpreted as graben, in impact basin structures. Both studies indicate higher thermal gradients on Ganymede than on Callisto in the period 3 to 4 billion years ago.

The detailed tectonics of grooved terrain formation on Ganymede continue to receive special study. M.T. Zuber (Brown University) estimated the displacement that has occurred between large units of cratered terrain on Ganymede under the assumption that concentric arcs of ring structures in different units were formed by a single event; different locations of the centres of curvature for different units suggest displacements of up to $40 \mathrm{deg}$ arc. M.P. Golombek (Lunar and Planetary Institute) derived a limit of one per cent on the increase in satellite radius associated with extension in the grooved terrain on the assumptions that the grooves are graben and that the dip angles of the bounding normal faults are $60^{\circ}$ or greater. This limit agrees with that of McKinnon ${ }^{11}$ based on the lack of tectonic disruption of Galileo
Regio. S.K. Croft (Lunar and Planetary Institute) proposed that grooved terrain is formed by hydraulic fracturing driven by overpressured water during the final phases of global freezing following early differentiation.

Many of the icy satellites of Saturn show extensional features broadly similar to those of Ganymede ${ }^{2}$. S.W. Squyres, R.T. Reynolds, P. Cassen (Ames Research Center) and S.J. Peale (University of California, Santa Barbara) drew particular attention to Enceladus, which shows features similar to the grooves of Ganymede and large areas of low crater density. Resurfacing of such younger areas could occur either by extrusion of fresh material or by complete relaxation of topography due to a high thermal gradient. A new model by D.J. Stevenson (Caltech) for magma migration by crack propagation through the lithosphere, when applied to Enceladus, suggests that volcanism may occur by comparatively infrequent eruptions of large volumes of $\mathrm{H}_{2} \mathrm{O}-\mathrm{NH}_{3}$ fluids, lending support to the volcanic resurfacing hypothesis. Squyres and his colleagues showed that tidal dissipation is an adequate heat source for volcanism on Enceladus as long as a forced eccentricity several times higher than the current value existed continuously or episodically through a substantial part of its history.

As the data from Pioneer, Venera and Voyager missions continue to be analysed, hypotheses for the evolution of Venus and of the outer planet satellites will be refined. To test these hypotheses will require new spacecraft observations. The US Galileo mission to Jupiter will help provide data for the jovian satellites, and the Soviet Union has announced plans for additional landers to be sent to the Venus surface to conduct further chemical analyses and imaging experiments. A major gap in our knowledge of Venus, however, is a global map of the surface at sufficiently fine resolution to distinguish among the proposed hypotheses for the tectonic evolution of the planet. While the proposed Venus Orbital Imaging Radar mission has fallen victim to reductions in the NASA planetary programme budget, a less ambitious and less costly Venus radar mapping mission is currently being considered to fill this gap. Completion of such a mission would provide a major step towards a full understanding of the evolution of Earth-like planets.

\section{Corrigendum}

In the News and Views article on 'Cause and treatment of atherosclerosis' by C.T. Dollery (Nature 297, 286; 1982), the sentence "C.T. Dollery (Royal Postgraduate Medical School, London) described a new method for measuring plasma 6-oxo-PGF, the hydrolysis product of $\mathrm{PGI}_{2}$, which uses gas chromatography-negative ion chemical ionization mass spectrometry and has a sensitivity limit of $500 \mathrm{pg} \mathrm{ml}^{-1}$ " should read ". . . a sensitivity limit of $500 \mathrm{cg} \mathrm{ml}^{-1}$ ", 